\title{
Pirfenidon plus Nintedanib bei idiopathischer pulmonaler Fibrose
}

Gesicherte Tatsachen

- Pirfenidon und Nintedanib sind zwei antifibrotische Wirkstoffe mit erwiesenem therapeutischen Nutzen (Aufhalten der Progression) bei Patienten mit idiopathischer pulmonaler Fibrose (IPF).

- Der therapeutische Nutzen einer konkomitanten Therapie bei IPF ist bisher nicht untersucht worden, und es liegen keine Daten zur Sicherheit und Verträglichkeit der gleichzeitigen Anwendung von Pirfenidon und Nintedanib bei kaukasischen Patienten vor.

Neue Aspekte

- Die konkomitante Behandlung mit Pirfenidon und Nintedanib scheint eine gangbare Therapieoption darzustellen, von der Patienten, die auf eine antifibrotische Monotherapie mit Pirfenidon nicht ansprechen, in erheblichem Maße profitieren könnten.

\section{Schlüsselwörter}

Pirfenidon · Nintedanib · Konkomitante Therapie .

Idiopathische pulmonale Fibrose

\section{Zusammenfassung}

Pirfenidon und Nintedanib sind pleiotrope antifibrotische Substanzen, die als Monotherapie zur Behandlung der idiopathischen pulmonalen Fibrose (IPF) zugelassen sind. Bisher liegt keine Evidenz für ihre Wirksamkeit in der gleichzeitigen Anwendung vor. Wir stellen in diesem Beitrag den ersten Fall eines kaukasischen Patienten mit IPF vor, der nach 2-jähriger Pirfenidon-Monotherapie mit Pirfenidon und Nintedanib gleichzeitig behandelt wurde. Über den 24-MonatsZeitraum war eine deutliche Abnahme der forcierten Vitalkapazität des Patienten zu verzeichnen, von 3,5 I vor Therapiebeginn auf 2,5 I

nach 24 Monaten. Im März 2015 wurde die konkomitante Gabe von Nintedanib eingeleitet. Die Lungenfunktion stabilisierte sich, und beide Mittel wurden gut vertragen. Die Behandlung mit Pirfenidon und Nintedanib dauert seit knapp 12 Monaten an. Dieser Beitrag der erste Bericht einer erfolgreichen Langzeittherapie mit Pirfenidon und Nintedanib - deutet darauf hin, dass eine konkomitante antifibrotische Therapie in ausgewählten Fällen eine sichere und therapeutisch wertvolle Eskalationsoption nach einer Pirfenidon-Monotherapie darstellen könnte. 


\section{Hintergrund}

Die idiopathische pulmonale Fibrose (IPF) ist immer noch eine unheilbare Krankheit, die zur respiratorischen Insuffizienz und zum Tod führt. Pirfenidon und Nintedanib sind die zwei einzigen zugelassenen Medikamente für die Behandlung von Patienten mit IPF.Pirfenidon dämpft dieFibroblastenproliferation, die Produktion von fibroseassoziierten Proteinen und Zytokinen sowie die erhöhte Biosynthese und Ansammlung von extrazellulärer Matrix als Reaktion auf Zytokin-Wachstumsfaktoren wie z.B. den transformierenden Wachstumsfaktor-beta (TGF- $\beta$ ) und den Plättchenwachstumsfaktor (PDGF). Nintedanib ist ein Tyrosinkinase-Inhibitor, der den vaskulären endothelialen Wachstumsfaktor (VEGF), den Fibroblasten-Wachstumsfaktor (FGF) und den PDGF hemmt [1].

In Phase-III-Studien wurde gezeigt, dass beide Medikamente jeweils und gleichermaßen zu einer Verlangsamung des Abfalls der forcierten Vitalkapazität (FVC) bei Patienten mit IPF führen [1-5]. Diese positive Wirkung der spezifischen antifibrotischen Medikamente ist leider nicht bei allen Patienten mit IPF nachweisbar und die Faktoren, die mit einer therapeutischen Ansprache assoziiert sind, sind noch nicht bekannt. Die häufigsten Nebenwirkungen Pirfenidons sind gastrointestinale Symptome und eine gesteigerte Lichtempfindlichkeit. Pirfenidon wird durch CYP1A2 metabolisiert, und Leberwertkontrollen sollten regelmäßig durchgeführt werden, um das Entstehen hepatotoxischer Nebenwirkungen frühzeitig zu erkennen. Häufige Nebenwirkungen bei Nintedanib sind Diarrhoe, Übelkeit, Gewichtsverlust und eine Erhöhung der Leberenzyme. Die Nebenwirkungen beider Medikamente sind meistens gut behandelbar, obwohl sie manchmal zum Absetzen der Therapie führen [1-5].

Angesichts der vergleichbaren Wirksamkeit existieren derzeit keine Richtlinien bezüglich der bevorzugt einzuleitenden antifibrotischen Therapie. Die Therapieentscheidung wird individuell angepasst und basiert auf dem funktionellen Status, den Komorbiditäten und den Präferenzen des Patienten sowie auf der Erfahrung des behandelnden Arztes mit den beiden Wirkstoffen [1].

Es ist auch noch nicht klar, ob eine Therapieumstellung bei lungenfunktioneller Verschlechterung unter einer Monothera- pie zu einer Stabilisierung der Krankheit führt. Ebenso gibt es bislang keine Erkenntnis darüber, ob die Kombination von Pirfenidon und Nintedanib bei Patienten mit IPF wirksamer ist als die Behandlung mit nur einem Medikament. In diesem Sinne bleibt die Behandlung der IPF eine Herausforderung.

\section{Studienergebnisse}

In ihrer Kasuistik beschreiben Hagmeyer et al. einen Patienten, der nach einer zweijährigen Behandlung mit Pirfenidon einen Abfall der FVC sowie der Diffusionskapazität aufwies. Unter der Therapie mit Pirfenidon war der Abfall der FVC initial recht rasch in den ersten Monaten (-19\% pro Jahr), später eher langsamer (-8,9\% pro Jahr). Nach einem Gespräch mit dem Patienten wurde entschieden, die Therapie mit Pirfenidon fortzuführen und zusätzlich eine Therapie mit Nintedanib zu beginnen. Die Dosierung beider Medikamente wurde initial reduziert (Pirfenidon: 1602 mg täglich, Nintedanib: 100 mg täglich). Die empfohlene Dosis von 2403 mg/d für Pirfenidon und 300 mg/d für Nintedanib wurde nach zwölf Wochen erreicht. Es traten keine wesentlichen Nebenwirkungen auf. Unter der Kombinationstherapie war der Abfall der FVC langsamer als unter Pirfenidon ( $-4,1 \%$ pro Jahr).

\section{Transfer in die Praxis}

Nach der Erstbeschreibung einer Umstellung von Pirfenidon auf Nintedanib bei einem Patienten mit IPF [6] und der Publikation einer randomisierten, kontrollierten Studie zur Verträglichkeit und Pharmakokinetik von zusätzlichem Nintedanib auf Pirfenidon bei 50 japanischen Patienten mit IPF [7] ist dies die erste Langzeitbeobachtung über 12 Monate bei einem IPF Patienten. Die Kombinationstherapie mit Pirfenidon und Nintedanib scheint verlockend zu sein, vor allem im Falle eines Progresses unter der Monotherapie. Es existieren aber derzeit keine Daten zur Wirksamkeit. In der o.g. japanischen Studie wurden die Pharmakokinetik und die Nebenwirkungen während einer kurzen gleichzeitigen Behandlung mit Pirfenidon und Nintedanib untersucht. Unter diesen Bedingungen fand sich eine etwas erhöhte Nebenwirkungsrate sowie ein leicht erniedrigter Spiegel von Nintedanib. Alle Nebenwirkungen waren mild oder moderat. 


\begin{abstract}
Aktuell werden in drei klinischen Studien, unter anderem auch in Deutschland, Patienten rekrutiert, mit dem Ziel, die Verträglichkeit einer Kombinationstherapie mit Pirfenidon und Nintedanib bei Patienten mit IPF zu untersuchen [8-10]. Außerdem sollte die übrige, nichtmedikamentöse Therapie für Patienten mit IPF (angepasste Sauerstofftherapie besonders bei Belastung, Ausschluss von Komorbiditäten, Rehabilitation, palliative Therapie und Lungentransplantation) weiter berücksichtigt werden.
\end{abstract}

\section{Fazit}

Die Behandlung von Patienten mit IPF stellt weiterhin eine Herausforderung dar. Weitere randomisierte, Placebo-kontrollierte Studien sind erforderlich, um fundierte Ergebnisse bezüglich der Verträglichkeit und Wirksamkeit einer kombinierten Therapie mit Pirfenidon und Nintedanib zu erzielen. Aus diesen Gründen sollten Patienten mit IPF vorzugsweise in diese klinischen Studien eingeschlossen werden und nicht außerhalb von Studien kombiniert behandelt werden.

\section{Referenzen}

Raghu G, Rochwerg B, Zhang Y, et al: An official ATS/ERS/JRS/ALAT Clinical Practice Guideline: treatment of idiopathic pulmonary fibrosis. An update of the 2011 clinical practice guideline. Am J Respir Crit Care Med 2015; 192:e3-e19.

2 King TE Jr, Bradford WZ, Castro-Bernardini S, et al:: A phase 3 trial of pirfenidone in patients with idiopathic pulmonary fibrosis. N Engl J Med 2014; 370:2083-2092.

-3 Noble PW, Albera C, Bradford WZ, et al.: Pirfenidone in patients with idiopathic pulmonary fibrosis (capacity): two randomised trials. Lancet 2011; 377:1760-1769.

4 Richeldi L, Costabel U, Selman M, et al.: Efficacy of a tyrosine kinase inhibitor in idiopathic pulmonary fibrosis. N Engl J Med 2011;365:1079-1087.

5 Richeldi L, du Bois RM, Raghu G, et al.; INPULSIS Triallnvestigators: Efficacy and safety of nintedanib in idiopathic pulmonary fibrosis. N Engl J Med 2014;370:2071-2082.

-6 Milger K, Kneidinger N, Neurohr C, et al.: Switching to nintedanib after discontinuation of pirfenidone due to adverse events in IPF. Eur Respir J 2015;46:1217-1221.

7 Ogura T, Taniguchi H, Azuma A, et al.: Safety and pharmacokinetics of nintedanib and pirfenidone in idiopathic pulmonary fibrosis. Eur Respir J 2015;45:1382-1392.

8 Safety and tolerability study of pirfenidone in combination with nintedanib in participants with idiopathic pulmonary fibrosis (IPF). https://clinicaltrials.gov/ct2/show/NCT02598193 (Zugriff 05.09.2016).

9 Safety, tolerability and PK of nintedanib in combination with pirfenidone in IPF. https://clinicaltrials.gov/ct2/show/NCT02579603 (Zugriff 05.09.2016).

10 A study to compare the amount of nintedanib and pirfenidone in the blood when nintedanib and pirfenidone are given separately or in combination. https://clinicaltrials.gov/ct2/show/NCT02606877 (Zugriff 05.09.2016).

Kontaktadresse: Dr. Fotios Drakopanagiotakis, Medizinische Klinik und Poliklinik II, Klinikstraße 33, 35392 Gießen, Deutschland 